\title{
Frontières
}

\section{De simples larmes ou de puissantes molécules pour nos yeux en détresse?}

\section{Diane Laflamme}

Volume 21, numéro 2, printemps 2009

Détresse psychique et antidépresseurs

URI : https://id.erudit.org/iderudit/039449ar

DOI : https://doi.org/10.7202/039449ar

Aller au sommaire du numéro

Éditeur(s)

Université du Québec à Montréal

ISSN

1180-3479 (imprimé)

1916-0976 (numérique)

Découvrir la revue

Citer ce document

Laflamme, D. (2009). De simples larmes ou de puissantes molécules pour nos yeux en détresse ? Frontières, 21(2), 3-6. https://doi.org/10.7202/039449ar d'utilisation que vous pouvez consulter en ligne.

https://apropos.erudit.org/fr/usagers/politique-dutilisation/ 


$\begin{array}{lllllllll}D & \text { I } & \text { T } & 0 & \text { R } & \text { I } & \text { A } & \text { L }\end{array}$

\title{
De simples larmes ou de puissantes molécules pour nos yeux en détresse?
}

\author{
J'AI LA TÊTE QUI ÉCLATE, \\ J'VOUDRAIS SEULEMENT DORMIR, \\ M'ÉTENDRE SUR L'ASPHALTE \\ ET ME LAISSER MOURIR. \\ LUC PLAMONDON, LE MONDE EST STONE. \\ POUR SUPPORTER \\ LE DIFFICILE \\ ET L'INUTILE \\ Y A L'TOUR DE L'ÎLE \\ QUARANTE-DEUX MILLES \\ DE CHOSES TRANQUILLES \\ POUR OUBLIER \\ GRANDE BLESSURE \\ DESSOUS L'ARMURE \\ ÉTÉ, HIVER \\ Y A L'TOUR DE L'ÎLE, \\ L'ÎLE D'ORLÉANS. \\ FÉLIX LECLERC, LE TOUR DE L'îLE.
}

Diane Laflamme, Ph. D., rédactrice en chef.

Se pourrait-il qu'au Québec ce soient nos chansonniers plutôt que nos érudits qui savent le mieux trouver les mots pour nous faire partager ce qu'ils ont appris à force de côtoyer de près le mal de vivre? À l'intérieur du vaste répertoire où la musique aide à dire notre mal-être, les extraits cités en exergue ont été choisis parce qu'ils proposent un même remède en deux versions opposées: quand la vie pèse trop lourd, soit fermer les yeux sur cet insupportable monde, soit les ouvrir plus grand encore afin d'habiter intensément le paysage.

Pour le premier remède, qui consiste à dormir malgré votre tête "qui éclate», vous pourriez vous présenter à la pharmacie du coin avec une prescription pour des somnifères ou quelque autre molécule de nos temps modernes. L'autre remède pour vos blessures «dessous l'armure " vous obligera à acheter un billet d'autobus - ou d'avion, qui sait - pour l'Île d'Orléans, près de la ville de Québec. Aucun des deux remèdes n'est garanti; si les auteurs-compositeurs qui les proposent confirment quelque chose en les formulant, ce serait plutôt que même les vedettes célèbres et adulées ne sont pas à l'abri des coups de déprime.

Faut-il fermer les yeux pour s'évader, ne serait-ce qu'un court moment, d'un monde qui nous agresse de toutes parts, ou pour s'extirper de l'enchaînement inexorable de nos propres pensées qui tournent en rond dans notre monde intérieur? Fermer les yeux pour en sortir, pour ne plus faire partie de tout ça!

$\mathrm{Ou}$, au contraire, faut-il ouvrir les yeux encore plus grand à travers nos larmes pour découvrir ce qui ne se laisse pas voir au premier regard, pour nous laisser fasciner par la vie qui chemine tranquillement mais résolument dans nos paysages, sans égard pour ce que nous nommons le difficile ou l'inutile?

D'où surgit notre détresse : d'en avoir trop vu... ou de ne pas avoir réussi à voir ce qui compte vraiment? Et si nous pouvions nous donner le moyen d'ouvrir des yeux devenus plus compétents? Peut-être pourrions-nous, en dopant notre regard grâce à de puissantes molécules, améliorer nos perceptions, notre capacité d'attention, notre humeur. 


\section{LES USAGES ÉLARGIS DES ANTIDÉPRESSEURS: POUR FERMER LES YEUX OU POUR LES OUVRIR ENCORE PLUS GRAND?}

Le mal de vivre et la déprime ne sont pas des préoccupations réservées aux chansonniers et à leur public. Pour y pallier, la liste des antidépresseurs que l'on dit être «de la nouvelle génération » est déjà longue ${ }^{1}$ et les chercheurs en sont même rendus aux métaanalyses pour comparer leur efficacité et leur acceptabilité du point de vue des patients (voir par exemple, Cipriani et al., 2009). En matière de politique sociale, nos gouvernants ont aussi noté que la consommation de médicaments psychotropes est en train d'atteindre des sommets. Les statistiques sont éloquentes: au Canada, les consultations médicales pour la dépression auraient augmenté de $60 \%$ entre 1995 et 2003; en 2002, les Canadiens se classaient parmi les plus grands consommateurs de médicaments psychotropes au monde; au cours de l'année 2000, 19,4\% des Québécois âgés de plus de 65 ans, soit presque un sur cinq, ont reçu au moins une ordonnance de benzodiazépines, un anxiolytique (CEST, 2009, p. 48). Ces données sont citées dans l'Avis intitulé Médicaments psychotropes et usages élargis: un regard éthique, publié récemment par la Commission de l'éthique de la science et de la technologie, un organisme-conseil auprès du gouvernement du Québec.

Il importe de trouver une aide appropriée pour soulager le désarroi et la détresse psychique qui rendent nécessaires pour plusieurs personnes ces consultations médicales et ces médicaments psychotropes. Les œuvres littéraires décrivent de façon plus éloquente que les statistiques le tragique de la situation. Dans son premier roman intitulé Borderline, la Québécoise Marie-Sissi Labrèche donne la parole à une enfant pour nous introduire sans ménagement dans l'univers insoutenable qui s'installe quand la dépression menace d'engloutir ses victimes:

Ma mère, j'ai toujours pensé qu'elle ne tenait pas à moi. J'ai toujours pensé que, parce qu'elle se réfugiait trop souvent quelque part dans sa tête où je n'avais pas accès, elle ne tenait pas à moi. Ma mère pouvait passer des semaines comme ça, dans sa tête, à me regarder avec ses yeux bleus braqués sur moi, sans expression, ses yeux remplis de dépression qui me rendaient malade. Des semaines ainsi, assise sur sa chaise berçante sans se bercer, à me regarder. Sans parler. Aucun mot. Le silence. Que le bruit du réfrigérateur, que le bruit du chauffe-eau du monsieur d'en haut, que le bruit des coquerelles qui courent sur mes feuilles à dessins éparpillées un peu partout dans la cuisine. Mais aucune parole réconfortante, rassurante sortant de sa bouche. Non. Et moi, assise par terre, à ses pieds, je lui racontais des histoires à l'aide de mes poupées ou de mes petits bonhommes Fisher Price; des histoires qu'elle ne comprenait même pas à cause de ses maudites hormones défectueuses, ses maudites hormones passées date (Labrèche, 2003, p. 19-20).

Qu'il s'agisse de personnages de roman ou du citoyen ordinaire, que le problème soit considéré comme une maladie mentale ou neurologique avérée ou que la détresse en cause relève, du moins en partie, du niveau sociétal, des problèmes de dépression d'une telle ampleur nécessitent l'intervention d'une équipe multidisciplinaire, bien au fait de toutes les ressources qui peuvent être mises à contribution.

Dans son Avis mentionné plus haut, la Commission de l'éthique de la science et de la technologie signale cependant que la hausse des diagnostics de certains troubles mentaux et neurologiques ainsi que celle des prescriptions de neuromédicaments ne peuvent s'appuyer uniquement sur des raisons médicales. Selon la Commission, plusieurs facteurs interviennent dans ces hausses, entre autres ce qu'elle appelle les « usages élargis» des médicaments psychotropes (CEST, 2009, p. 138). Elle distingue entre les usages élargis de provenance «Médicale» et ceux qui sont liés au «Mode de vie $»^{2}$. Nous retiendrons cette classification pour notre propos, en nous intéressant surtout aux usages élargis de type Mode de vie des psychotropes ${ }^{3}$ et des antidépresseurs ${ }^{4}$, comme les définit la Commission.

\section{LES USAGES ÉLARGIS DE TYPE MODE DE VIE}

Les usages élargis de type Mode de vie désignent des usages visant à améliorer la performance ou encore à gérer des troubles de l'humeur ou des situations personnelles difficiles, même en l'absence de visée thérapeutique (CEST, 2009, chapitre 3). Les antidépresseurs, par exemple, ne seront plus réservés aux situations de dépression majeure: «Dorénavant, ces médicaments sont aussi utilisés pour les troubles anxieux, les douleurs neuropathiques (liées au SNC et aux nerfs), l'arrêt du tabagisme, les syndromes prémenstruels, de même que pour des usages non reconnus - et en expansion - lors de troubles de sommeil, de migraines et de fibromyalgie» (p. 83).

On voudra aussi recourir aux médicaments psychotropes pour favoriser un style de vie ainsi que les valeurs qui $\mathrm{y}$ sont sous-jacentes ou pour répondre à des attentes sociales liées à la performance. La Commission ne manque pas de signaler que ce sont nos valeurs qui sont alors mises en cause en même temps que nos choix en tant que consommateurs de médicaments: «plusieurs valeurs sont touchées par les usages élargis de neuromédicaments, tant des valeurs individuelles - telles que l'autonomie, le bien-être, la confiance en soi, la performance et la sécurité - que des valeurs collectives comme la solidarité et l'accessibilité» (p. 139).

L'Avis de la Commission porte bien son titre, c'est un «regard» qui se pose et qui nous oblige à nous demander jusqu'à quel point nous considérons qu'il est normal d'avoir à vivre des moments de détresse psychique. Ce regard demande aussi à son interlocuteur de lui répondre en déclinant son identité: «indépendamment des catégories, l'utilisation accrue de médicaments psychotropes pour des usages élargis pose la question de l'identité de la personne et de la normalité des comportements socialement attendus » (p. 96). En s'introduisant ainsi dans notre mode de vie, les médicaments psychotropes nous obligent à repenser notre capacité d'améliorer qui nous sommes et comment nous le devenons. 


\section{AMÉLIORER NOTRE CAPACITÉ}

\section{DE FAIRE LE TRAVAIL QUI CONSISTE À «ÊTRE »}

Loin de se contenter de construire des catégories pour classer les comportements observés, la Commission d'éthique de la science et de la technologie consacre de vastes sections des chapitres de son Avis à une réflexion sur la personne humaine et sur les représentations que nous nous en faisons individuellement et collectivement. Le débat sur les usages élargis des antidépresseurs se déplace vers une réflexion sur notre capacité d'advenir à nousmêmes et de construire notre identité. Comment pourrions-nous refuser de telles possibilités d'améliorer qui nous sommes? «La volonté de s'améliorer fait partie de la condition humaine, et cela inclut l'amélioration des fonctions cognitives. Ainsi, la question de l'amélioration par des médicaments psychotropes suscite des débats sur les visions que l'on entretient de l'être humain, de la vie réussie et de la réalisation de soi » (p. 128).

Dans le cadre d'un usage à visée améliorante ${ }^{5}$, les médicaments psychotropes viendront-ils alimenter notre capacité de faire ce travail spécifiquement humain qui consiste à «être », à construire notre propre identité et à inventer d'une génération à l'autre des comportements compatibles avec une vie sociale satisfaisante? Ne serionsnous pas plutôt à la recherche d'une solution à peu de frais pour alléger cette tâche, pour nous exempter du travail artisanal qu'est la réalisation de soi, un travail frustrant qui nous plonge trop souvent dans la détresse?

Et si cette vulnérabilité à la détresse était inséparable de ce qui fait de nous des êtres humains? Je pense ici à la polarité Humain / Déshumain, que Pierre Fédida a si bien nommée lorsqu'il en a fait le titre d'un ouvrage collectif publié en 2007. Sans cette vulnérabilité à la détresse, serionsnous encore humains? Fédida décrit notre façon d'advenir à nous-mêmes comme un double mouvement d'identification et de désidentification propre à l'humain:

Pourrait-on supposer qu'il y ait identi-

fication sans clivage? Non, l'identifica-

tion suppose le clivage du Moi. Freud

$\mathrm{y}$ tient jusqu'à la fin de sa vie, puisque,

au fond c'est la grande découverte de la

psychanalyse: reconnaître que nous ne

sommes pas un moi synthétique, que

nous sommes nécessairement en deux, ou deux. Quelle que soit la tragédie, ce qu'on peut appeler «humain» met en jeu la souffrance de ce clivage. Et, dans cette perspective, penser l'identification par rapport au clivage, c'est penser précisément que l'identification suppose constamment le mouvement de désidentification (Fédida, 2007, p. 93).

Et pour alimenter ce mouvement d'identification / désidentification, Fédida ose même nommer un phénomène inquiétant, qu'il présente comme «l'œuvre civilisatrice de la névrose»:

Il n'y aurait pas de possibilité d'identification s'il n'y avait pas l'œuvre civilisatrice de la névrose. La névrose accomplit une œuvre civilisatrice et on sait précisément qu'il faut concevoir une analyse non pas comme la suppression de la névrose, mais comme une façon de rendre la névrose plus maladive, plus efficace, plus perspicace, plus intelligente. Gardons surtout la névrose, gardons notre névrose, rendons-la simplement plus intelligente (Fédida, 2007, p. 93-94).
Les psychotropes et les antidépresseurs, dans leurs usages élargis de types Mode de vie, en viendront-ils à faire une œuvre civilisatrice? Mais pour rendre notre névrose plus intelligente, encore devrons-nous accepter de la garder et non tenter de nous en débarrasser à tout prix!

L'Avis de la Commission de l'éthique de la science et de la technologie énumère soigneusement les bénéfices, risques et dérives possibles des usages élargis de type Mode de vie des médicaments psychotropes. Parmi les bénéfices espérés: «l'avancée des connaissances, l'augmentation des fonctions cognitives, la régularisation de l'humeur et l'amélioration du niveau de vie personnel et sociétal» (p. 110). L'Avis de la Commissions se fait particulièrement éloquent à ce sujet:

En somme, les bénéfices découlant des usages élargis de presque toutes les classes de psychotropes laissent espérer une estime de soi plus forte, une reconnaissance extérieure des résultats par une promotion professionnelle, une vie sociale enrichie, une réputation avantageuse, etc. Il est possible que la somme de ces gains individuels tende à hausser les niveaux d'éducation et de productivité de la société, et que tous profiteraient de cette situation (CEST, 2009, p. XXIX).

$\mathrm{Au}$ nombre des risques: "les risques pour la sécurité des personnes, les pressions externes, la dépendance psychologique et la banalisation du psychotrope ». Dans sa conclusion, la Commission décrit trois dérives potentielles (p. XLVI) : Trois dérives potentielles des usages élargis de psychotropes retiennent l'attention de la Commission. La première ¿ consiste en la présence de pressions extérieures à l'individu et d'une information partielle, voire partiale, relative x aux psychotropes et à leurs bénéfices. La Commission y voit le risque d'une homogénéisation des humeurs et des comportements et, par conséquent, la démonstration d'une intolérance envers les individus qui dérogent d'une «normalité» dorénavant médicamentée. La deuxième dérive est liée à la banalisation des recours aux usages élargis «Mode de vie », et dans une moindre mesure «Médicaux». Certains usages en vue d'atteindre des objectifs personnels et professionnels, ou encore de faire face à des événements de vie, douloureux mais inévitables, marquent un glissement vers la maximisation des résultats rapides et la minimisation des ressources personnelles. Finalement, la troisième dérive met en cause la notion d'identité personnelle et la représentation de l'être humain. La réalisation de soi intègre la réussite et la performance, mais tous les moyens pour y parvenir sont-ils souhaitables?

En comptabilité double avec actif et passif, tel est le panorama qui s'offre à nos yeux. Les larmes feront place à l'étonnement, peut-être, devant tant de nouvelles ressources pour faire échec à la détresse et même pour rendre notre névrose plus intelligente.

Parmi les dérives possibles répertoriées par la Commission, je retiens le passage de la deuxième où il est question de minimiser nos capacités personnelles. Ouvrir ou fermer les yeux «sous influence », par exemple grâce à un médicament antidépresseur, 
est-ce une façon de se créer des chaînes ou des compétences? Nos yeux guéris de leurs larmes seront-ils aussi des yeux enchaînés? Si c'est le cas, saurons-nous ensuite trouver la capacité de briser ces nouvelles chaînes?

\section{PRENDRE LE RISQUE DE SE RETROUVER EN LARMES}

Le magnifique travail iconographique de Suzelle Levasseur qui accompagne les textes de ce numéro de Frontières s'intitule Lacrima. Il nous invite à redécouvrir cette étrange capacité que nous avons de nous mettre en larmes. Quand nous nous regardons aller, nous voyons bien que nous sommes plus empêtrés et impuissants que triomphants dans le travail humain qui consiste tout simplement à "être». Cette capacité de voir - d'ouvrir et de fermer les yeux pour en prendre et pour en laisser - nous rend éminemment vulnérables à des moments de désarroi et de détresse psychique. Être vulnérable à des coups de déprime, à des épisodes de mal-être ne constitue pas une tare mais notre lot commun; comment pourrions-nous l'oublier?

La complexité de nos systèmes sociaux fait en sorte que dans tous les domaines qui comptent pour une vie réussie, nous devons maintenant faire appel à des outils plus complexes et à des conseillers plus spécialisés afin d'évaluer intelligemment quel rapport risques-bénéfices nous convient le mieux pour gérer nos avoirs. À mesure que nous aurons accès aux plus-values personnelles et sociales que laissent entrevoir les usages élargis de type Mode de vie des médicaments psychotropes, il nous faudra apprendre à faire ce calcul risques-bénéfices ${ }^{6}$ dans un contexte plus riche en possibilités pour notre capacité "d'être».

Quant au privilège qui consiste à être capable de verser des larmes, c'est peut-être maintenant qu'il nous est le plus précieux.

\section{Bibliographie}

CIPRIANI, A., T.A. FURUKAWA, G. SALANTI, J.R. GEDDES, J.P. HIGGINS, R. CHURCHILL et al. (2009). "Comparative efficacy and acceptability of 12 new-generation antidepressants: A multipletreatments meta-analysis », The Lancet, vol. 373, n 9665, le 28 février, p. 746-758.

COMMISSION DE L'ÉTHIQUE DE LA SCIENCE ET DE LA TECHNOLOGIE (CEST) (2009). Avis. Médicaments psychotropes et usages élargis: un regard éthique, en ligne, < http://www. ethique.gouv.qc.ca/index.php ?option $=$ com_docman\&task $=\mathrm{doc}_{-}$ download\&gid=93\&Itemid $=108>$, Gouvernement du Québec, consulté le 2009-06-12.

FÉDIDA, P. (dir.) (2007). Humain / Déshumain, Paris, PUF.

LABRÈCHE, M.-S. (2003). Borderline, Montréal, Boréal.

\section{Notes}

1. Une simple énumération des noms de ces antidépresseurs suffit pour nous faire mesurer la distance entre le vocabulaire des neuromédicaments et celui de la chanson et de la poésie: bupropion, citalopram, duloxétine, escitalopram, fluoxétine, fluvoxamine, milnacipran, mirtazapin, paroxétine, réboxétine, sertraline, venlafaxine. Ces douze antidépresseurs font l'objet de la méta-analyse de Cipriani et al. (2009).

2. "Dans cet avis, la Commission privilégie le terme "usage élargi" entendu comme un usage en dehors des pratiques établies et combinant les dimensions médicale et sociale» (CEST, 2009, p. 82). "Dans son avis, la Commission considère deux grandes catégories d'usages élargis, soit ceux de nature "Médicale" et ceux qui participent davantage d'un "Mode de vie". La première relève de la sphère d'expertise des médecins qui effectuent l'activité réservée de prescrire les médicaments pour traiter des maladies et sont donc en mesure de légitimer leur utilisation. La seconde catégorie concerne l'utilisation des neuromédicaments pour satisfaire un style de vie» (CEST, 2009, p. 110).

3. La CEST retient les définitions suivantes pour présenter les psychotropes, les médicaments psychotropes et leurs applications : «Il n'existe pas de définition universelle de psychotrope. Pour l'Office québécois de la langue française, un psychotrope est une "substance d'origine naturelle ou artificielle capable de modifier l'activité mentale et dont l'action essentielle s'exerce sur le système nerveux central et le psychisme". Sa définition propose aussi comme synonymes "substance psychotrope" et "médicament psychotrope". Ainsi, les psychotropes ne se limitent pas aux médicaments prescrits mais incluent toute substance qui influence le SNC (système nerveux central). La caféine, la nicotine, la cocaïne et l'alcool sont donc des psychotropes. Dans cet avis, la Commission [...] retient comme définition de "psychotrope" celle d'un médicament d'ordonnance ayant un effet sur le système nerveux central et sur le psychisme. Dans l'avis, les termes médicament psychotrope et neuromédicament seront utilisés comme synonymes de "psychotrope" »(CEST, 2009, p. 6). «Les applications des médicaments psychotropes: Les psychotropes modifient la neurochimie et les communications électriques du cerveau. Ils sont employés dans le traitement de la douleur, des troubles cognitifs (touchant notamment la mémoire, l'attention, la vigilance, la concentration et la résolution de problèmes), des troubles de l'humeur (comme la dépression et la bipolarité), des troubles anxieux (comme l'anxiété et les phobies), des troubles de comportement, des troubles neurologiques (dont le TDA/H) et des troubles de la personnalité (comme la personnalité limite et le trouble obsessionnel-compulsif). Les neuromédicaments agissent principalement sur le niveau de concentration des neurotransmetteurs, en modifiant leur action ou leur durée d'action» (CEST, 2009, p. 50).

4. La CEST retient les définitions suivantes pour présenter les antidépresseurs ainsi que les bénéfices, risques et effets indésirables qui s'y rattachent: "Les antidépresseurs sont des médicaments qui traitent des désordres affectifs comme la dépression majeure. L'arrivée d'une nouvelle génération d'antidépresseurs, les inhibiteurs sélectifs de la recapture de la sérotonine (ISRS, ou SSRI en anglais), a modifié les possibilités de traitement médicamenteux. En effet, ces antidépresseurs ont nettement moins d'effets secondaires indésirables que les précédents (comme les antidépresseurs tricycliques) et leur spectre d'action est moins large car ils ciblent spécifiquement certains neurotransmetteurs (CEST, 2009, p. 51). Bénéfices: "Antidépresseurs: diminution des symptômes dépressifs, diminution du stress et de l'anxiété, diminution des idées suicidaires, amélioration du sommeil, amélioration des tâches scolaires et professionnelles, augmentation de la confiance et de l'estime de soi, augmentation des contacts et comportements sociaux positifs » (CEST, 2009, p. 68). Risques et effets indésirables: "Antidépresseurs: modification du rythme cardiaque (dont la tachycardie et l'arythmie, surtout avec les antidépresseurs tricycliques), risques accrus de suicide chez les 0-24 ans (pour les ISRS), difficultés respiratoires, céphalées, nausées, problèmes digestifs, somnolence, insomnie, troubles psychomoteurs, étourdissements, gain et / ou perte de poids, irritabilité, dysfonctions sexuelles, bouche sèche, agitation» (CEST, 2009, p. 69).

5. La Commission précise comme suit la distinction entre amélioration et thérapie: "La littérature anglophone utilise le terme anglais enhancement [...] Enhancement se traduit toutefois mal en français. "Amélioration" est le plus souvent utilisé, mais le mot anglais inclut les idées de perfectibilité et de performance qui ne sont pas clairement reprises dans la traduction usuelle. Des suggestions peuvent être faites, comme amélioration des potentialités, rehaussement des potentialités, amélioration des performances, augmentation des performances, mais le débat reste à tenir. Dans son avis, la Commission utilise le mot "amélioration" dans un sens qui intègre les notions de performance et de perfectibilité de l'individu. Elle note aussi que cette absence de correspondance "parfaite" ajoute encore à la difficulté des débats entourant les mots "amélioration" et "thérapie" » (CEST, 2009, p. 88).

6. «Le rapport "risques / bénéfices" soupèse, dans une situation précise, les risques possibles et les bénéfices espérés associés à la prise d'un médicament précis. Il vise à atteindre l'usage optimal, qui se définit comme "l'usage qui maximise les bienfaits et minimise les risques pour la santé de la population en tenant compte des diverses options possibles, des coûts et des ressources disponibles, des valeurs des patients et des valeurs sociales". L'évaluation de ce rapport se base sur les données probantes, les guides de bonnes pratiques, les lignes directrices et l'expérience clinique (ou le jugement professionnel). À une situation donnée, c'est-à-dire des indications pour lesquelles un médicament précis est approuvé, s'ajoute le contexte particulier de la consultation médicale et du positionnement du clinicien par rapport aux psychotropes. Ainsi, pour le même trouble de santé suspecté, le rapport "risques / bénéfices" va varier en fonction du médecin, de l'individu qui consulte, de son état de santé général, de sa médication actuelle et de ses antécédents médicaux» (CEST, 2009, p. 68). 